\title{
Large scale dynamos with helicity loss through boundaries
}

\author{
A. Brandenburg` and W. Dobler \\ Department of Mathematics, University of Newcastle upon Tyne, NE1 7RU, UK \\ Received 22 December 2000 / Accepted 22 January 2001

\begin{abstract}
Dynamo action is investigated in simulations of locally isotropic and homogeneous turbulence in a slab between open boundaries. It is found that a "pseudo-vacuum" boundary condition (where the field is vertical) leads to strong helicity fluxes which significantly reduce the amplitude of the resulting large-scale field. On the other hand, if there is a conducting halo outside the dynamo-active region, the large scale field amplitude can reach larger values, but the time scale after which this field is reached increases linearly with the magnetic Reynolds number. In both cases, most of the helicity flux is found to occur on large scales. From the variety of models considered we conclude that open boundaries tend to lower the saturation field strength compared to the case with periodic boundaries. The rate at which this lower saturation field strength is attained is roughly independent of the strength of the turbulence and of the boundary conditions. For dynamos with less helicity, however, significant field strengths could be reached in a shorter time.
\end{abstract}

Key words. MHD - turbulence

\section{Introduction}

Significant progress has been made in recent years in the understanding of the generation of large-scale magnetic fields in astrophysical bodies by dynamo action. Some essential features of mean-field dynamo theory (e.g., Moffatt 1978; Krause \& Rädler 1980) are recovered qualitatively and quantitatively in direct three-dimensional simulations, but the time scale on which large-scale fields are established becomes very long as the magnetic Reynolds number increases (Brandenburg 2001a, hereafter referred to as B2001). This result, which has been obtained using simulations of helical turbulence in a periodic domain, is now understood to be a consequence of the fact that the large-scale magnetic fields generated by such flows possess magnetic helicity which is conserved, except for (microscopic) resistive effects. This behaviour can also be reproduced with a mean-field model with resistively dominated ("catastrophic") quenching of $\alpha$-effect and turbulent magnetic diffusivity; see B2001 for details. The implications of boundary conditions for "catastrophic" $\alpha$ quenching were first pointed out by Blackman \& Field (2000a), who showed that the results of earlier simulations by Cattaneo \& Hughes (1996) could be understood as a consequence of periodic boundary conditions.

Send offprint requests to: A. Brandenburg,

e-mail: brandenb@nordita.dk

* Also at: NORDITA, Blegdamsvej 17, 2100 Copenhagen $\varnothing$, Denmark.
The problem is that in order to generate large-scale fields with finite magnetic helicity on time scales shorter than the resistive time, one also needs to generate magnetic fields with the opposite sign of magnetic helicity, so that the net magnetic helicity stays close to its initial value. In an astrophysical body, the sign of the magnetic helicity is different in the two hemispheres, but preliminary models with helicity flux through the equatorial plane suggest that this actually diminishes the net large-scale magnetic field rather than enhancing it (Brandenburg 2001b). The other possibility is that one can have a segregation in scales (e.g. Seehafer 1996) such that large-scale magnetic fields with magnetic helicity of one sign are generated within the domain of interest, whilst small-scale magnetic fields with opposite sign of magnetic helicity are expelled through the boundaries (Blackman \& Field 2000b). Whether or not this mechanism really works is unclear. So far there has only been a phenomenological approach suggesting that the $\alpha$-effect would be large if a flux of magnetic helicity through the boundaries was possible (Kleeorin et al. 2000).

The aim of the present paper is to investigate the effect of open boundaries on the dynamo. A simple local boundary condition is the vertical field condition (sometimes also referred to as pseudo-vacuum boundary condition), that was used previously both in magnetoconvection (Hurlburt \& Toomre 1988) and in accretion disc simulations showing dynamo action (Brandenburg et al. 1995). This boundary condition allows for a finite magnetic helicity flux through the boundaries, even though the flux of magnetic energy 
(i.e. the Poynting flux) is still zero. We also consider cases with a conducting halo outside the disc, as well as cases with density stratification, so that magnetic buoyancy can contribute to turbulent flux transport out of the dynamoactive region.

\section{The model}

We solve the isothermal compressible MHD equations for the logarithmic density $\ln \rho$, the velocity $\boldsymbol{u}$, and the magnetic vector potential $\boldsymbol{A}$,

$\frac{\mathrm{D} \ln \rho}{\mathrm{D} t}=-\boldsymbol{\nabla} \cdot \boldsymbol{u}$

$\frac{\mathrm{D} \boldsymbol{u}}{\mathrm{D} t}=-c_{\mathrm{s}}^{2} \boldsymbol{\nabla} \ln \rho+\frac{\boldsymbol{J} \times \boldsymbol{B}}{\rho}+\frac{\mu}{\rho}\left(\nabla^{2} \boldsymbol{u}+\frac{1}{3} \nabla \boldsymbol{\nabla} \cdot \boldsymbol{u}\right)+\boldsymbol{f},(2)$

$\frac{\partial \boldsymbol{A}}{\partial t}=\boldsymbol{u} \times \boldsymbol{B}-\eta \mu_{0} \boldsymbol{J}$

where $\mathrm{D} / \mathrm{D} t=\partial / \partial t+\boldsymbol{u} \cdot \boldsymbol{\nabla}$ is the advective derivative, $\boldsymbol{B}=\boldsymbol{\nabla} \times \boldsymbol{A}$ the magnetic field, $\boldsymbol{J}=\boldsymbol{\nabla} \times \boldsymbol{B} / \mu_{0}$ the current density, and $\boldsymbol{f}$ is the random forcing function specified in B2001. The induction Eq. (3) implies a specific gauge for $\boldsymbol{A}$; in Sect. 4 we discuss how to calculate gaugeindependent magnetic helicity and helicity fluxes. Instead of the dynamical viscosity $\mu$ (= const) we will in the following refer to $\nu \equiv \mu / \rho_{0}$, where $\rho_{0}$ is the mean density in the domain ( $\rho_{0}=$ const owing to mass conservation). We use nondimensional units where $c_{\mathrm{s}}=k_{1}=\rho_{0}=\mu_{0}=1$. Here, $c_{\mathrm{s}}=$ const is the sound speed, $k_{1}$ the smallest wavenumber of the domain (so its size is $2 \pi$ ), and $\mu_{0}$ is the vacuum permeability.

We use sixth-order finite difference and a third-order time stepping scheme. The number of meshpoints is usually $120^{3}$, except for a few low Reynolds number runs.

All variables are assumed to be periodic in the $x$ - and $y$-directions. This implies that the vertical magnetic flux vanishes, because $B_{z}=\partial_{x} A_{y}-\partial_{y} A_{x}$, so the integral of $B_{z}$ over $x$ and $y$ vanishes. Therefore the horizontal average of the vertical field also vanishes. In the $z$-direction we assume either also periodicity (if there is a halo), or we adopt a stress-free boundary condition where the field is purely vertical (if there is no halo). The latter states that $\partial_{z} u_{x}=\partial_{z} u_{y}=u_{z}=0$ together with $B_{x}=B_{y}=0$, and solenoidality then implies that $\partial_{z} B_{z}=0$ on the vertical boundaries. In terms of $\boldsymbol{A}$, this boundary condition is $\partial_{z} A_{x}=\partial_{z} A_{y}=A_{z}=0$. In models with a halo the extent in the $z$-direction is $4 \pi$ and $\boldsymbol{f}$ is multiplied by a mask function

$p(z)=\left\{\begin{array}{lll}\exp \left[-z^{2} /\left(H^{2}-z^{2}\right)\right] & \text { for } & |z|<H, \\ 0 & \text { for } & |z| \geq H,\end{array}\right.$

where $H=\pi$ is the semi-height of the disc. In the models with halo we adopt periodic boundary conditions which has some conceptual advantages in that all the magnetic energy and helicity that crosses the boundary at $z= \pm H$ can be accounted for. We also consider models
Table 1. Summary of the main properties of the runs with vertical field boundary condition and with conducting halo. The models Buoy 1 and Buoy 2 are stratified and allow for magnetic buoyancy effects. The parameter $q=\left\langle\overline{\boldsymbol{B}}^{2}\right\rangle /\left\langle\boldsymbol{B}^{2}\right\rangle$ gives the fractional magnetic energy in the mean field relative to the total magnetic field

\begin{tabular}{ccccccc}
\hline & $\nu$ & $\eta$ & $\eta_{\text {halo }}$ & $u_{\text {rms }}$ & $b_{\text {rms }}$ & $q$ \\
\hline Vert 1 & 0.01 & 0.01 & - & 0.100 & 0.093 & 0.76 \\
Vert 2 & 0.005 & 0.005 & - & 0.161 & 0.141 & 0.59 \\
Vert 3 & 0.002 & 0.002 & - & 0.197 & 0.178 & 0.39 \\
Vert 4 & 0.002 & 0.001 & - & 0.191 & 0.166 & 0.24 \\
Halo 1 & 0.002 & 0.0005 & 0.02 & 0.116 & 0.137 & 0.44 \\
Halo 2 & 0.002 & 0.001 & 0.02 & 0.118 & 0.156 & 0.60 \\
Buoy 1 & 0.002 & 0.001 & 0.02 & 0.124 & 0.200 & 0.61 \\
Buoy 2 & 0.002 & 0.001 & 0.001 & 0.147 & 0.242 & 0.71 \\
\hline
\end{tabular}

where, in addition to the halo, we have imposed gravitational acceleration, so $\boldsymbol{f} \rightarrow \boldsymbol{f}+\boldsymbol{g}$, where $\boldsymbol{g}=-\boldsymbol{\nabla} \Phi$ and $\Phi=-g_{0} \cos (z / 2)$ is a periodic gravitational potential in $|z|<2 \pi$. This produces a density enhancement near the midplane at $z=0$. The models presented below have $g_{0}=0.5$, so the maximum density contrast is $\Delta \ln \rho=2 g_{0} / c_{\mathrm{s}}^{2}=1$. These models allow for additional flux loss via magnetic buoyancy. We first discuss the models without halo and uniform background density.

\section{Description of the various runs}

The parameters of the various runs are summarized in Table 1. All runs develop a large-scale magnetic field after some time. This large-scale field points in the horizontal directions and varies along $z$. However, in some cases there can be intermediate stages during which the large scale field is oriented differently. The different orientations of the mean field are best described by calculating the three different mean fields, whose energies are denoted in the following by $K_{x}, K_{y}$, and $K_{z}$. The corresponding mean fields vary only in the $x, y$, and $z$ directions, respectively, and are averaged in the two perpendicular directions; see also Eqs. (8)-(10) of B2001. The mean field that varies only in the $z$-direction, and which is averaged over $x$ and $y$, is denoted in the following by an overbar.

\subsection{Runs with vertical field boundary condition}

In Fig. 1 we show the resulting field structure near the end of run Vert 4 at $t=1150$. Unlike the case with periodic boundaries where the presence of a large scale field was readily visible in two-dimensional slices of individual field components (see Fig. 4 in B2001), averaging over one of the two horizontal coordinate directions is required before the mean field becomes visible.

Averaging over both horizontal coordinate directions clearly yields the profiles of the two non-vanishing field components, $\bar{B}_{x}$ and $\bar{B}_{y}$; see Fig. 2 . Note that one of the two components (here $\bar{B}_{x}$ ) is approximately 

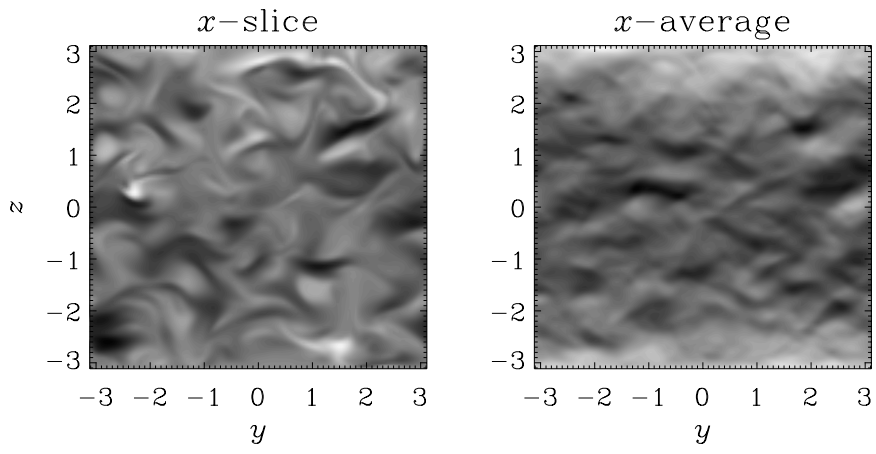

Fig. 1. Images of a cross-section and an $x$-average of $B_{y}$. Vert $4, t=1150$. Dark and light indicate shades indicate negative and positive values, respectively. Note the high noise level in the cross-section

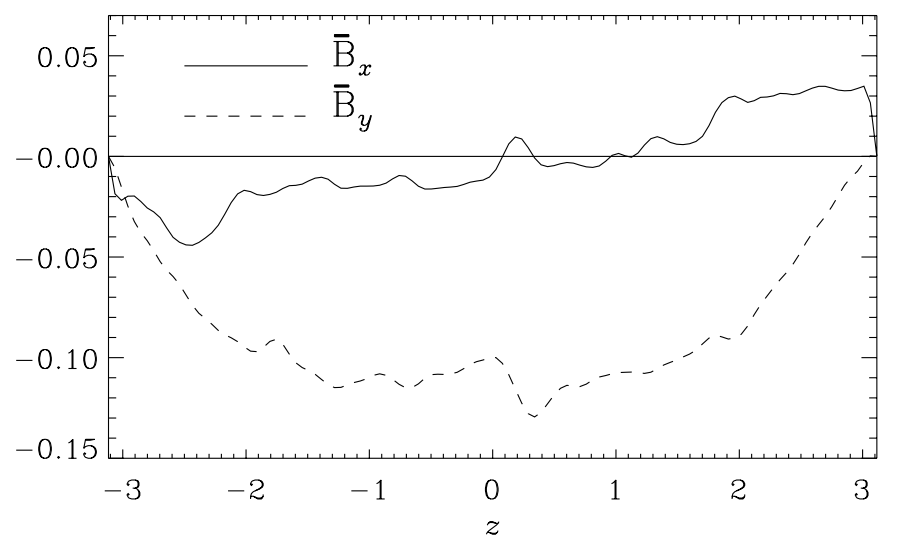

Fig. 2. Horizontal averages of $B_{x}$ and $B_{y}$. Vert 4

antisymmetric about the midplane, whilst the other (here $\bar{B}_{y}$ ) is approximately symmetric about the midplane and of larger amplitude. There are also some indications of boundary layer behaviour which was seen in highly nonlinear $\alpha^{2}$-dynamos (Meinel \& Brandenburg 1990), with the same boundary conditions as in the present simulations, i.e. $\bar{B}_{x}=\bar{B}_{y}=0$.

In Fig. 3 we plot the evolution of the magnetic energy within the domain. The magnetic diffusion time is $\left(\eta k_{1}^{2}\right)^{-1} \approx 500$. For $t \lesssim 200$, magnetic energy is mainly in the small scales, for which the helicity constraint is irrelevant, so the initial exponential growth can occur on a dynamical time scale. At $t=400$, the magnetic energy reaches a peak. At that time most of the large scale field energy is actually in $K_{y}$, which denotes the energy of the mean field defined by averaging in the two directions perpendicular to $y$, so this field varies only in the $y$-direction. This field is different from the anticipated field $K_{z}$ that is obtained by averaging in the $x$ and $y$-directions. This $K_{z}$ field also builds up and saturates around $t=700$, but it reaches a saturation level that is significantly less than in the case of periodic domains (B2001). Furthermore, as the magnetic Reynolds number increases, the fractional energy of the mean field relative to the total magnetic field decreases; see Fig. 4.

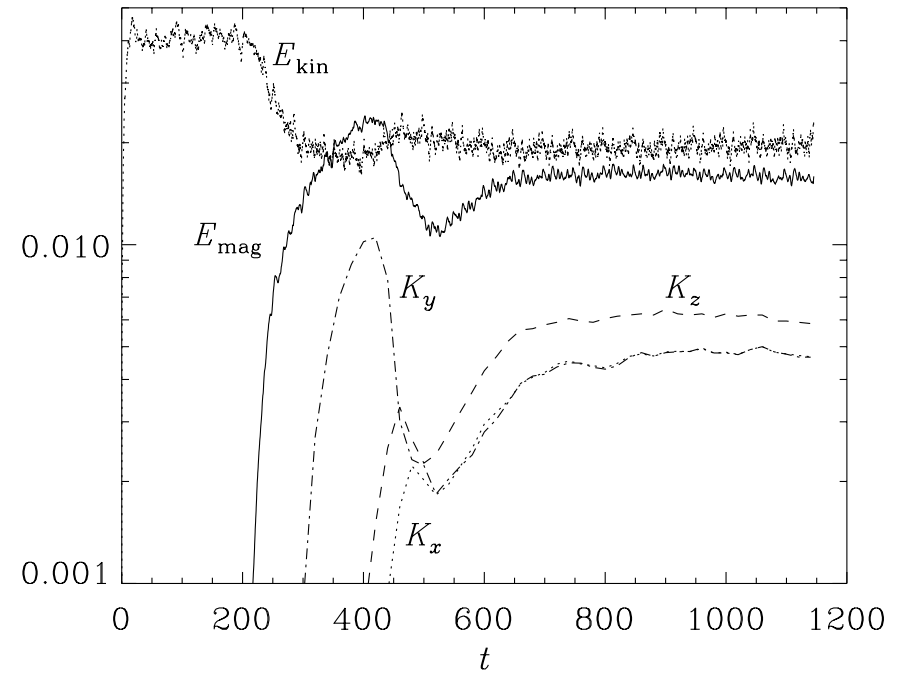

Fig. 3. Evolution of kinetic and magnetic energy together with the magnetic energies contained in three differently averaged mean fields. Vert 3

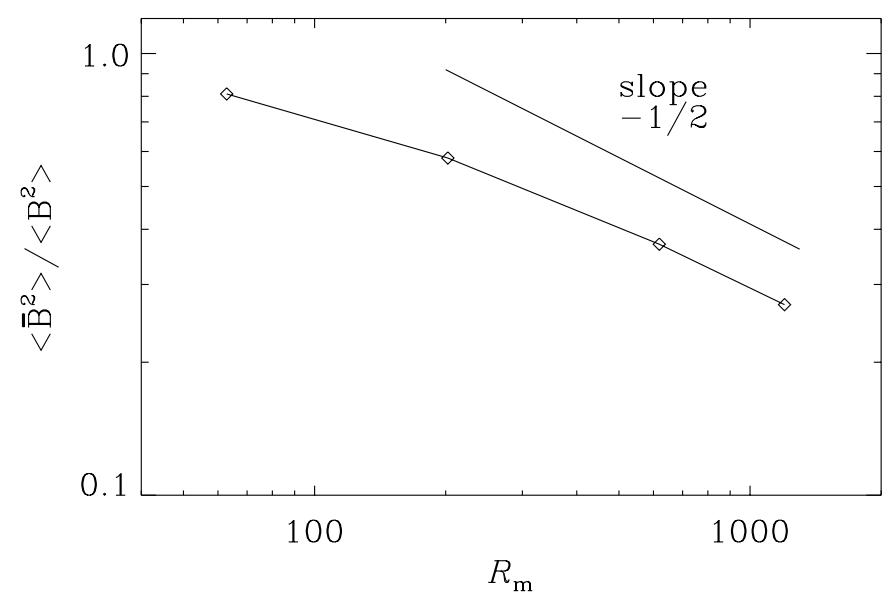

Fig. 4. Mean-field magnetic energy relative to the total magnetic energy, $q=\left\langle\overline{\boldsymbol{B}}^{2}\right\rangle /\left\langle\boldsymbol{B}^{2}\right\rangle$, for different values of the magnetic Reynolds number, $R_{\mathrm{m}}=u_{\mathrm{rms}} L / \eta$, where $L=2 \pi$ is the scale of the simulation domain

\subsection{Runs with a conducting halo}

We have carried out runs with low and high halo conductivities, with and without imposed gravity. All these runs are similar with respect to the overall field evolution in that they all produce a clearly visible large scale field that grows slowly (on a resistive time scale), reaching finally super-equipartition field strengths. These runs are therefore similar to those of periodic domains, but rather different to those with vertical field boundary conditions.

In Fig. 5 we show a vertical slice of the resulting field structure for a run with gravity $\left(g_{0}=0.5\right)$ and a halo conductivity that is equal to the conductivity in the turbulent zone (in $|z|<\pi$ ). Note that, in contrast to Fig. 1, the large scale field can clearly be seen even without averaging. The horizontally averaged field components are shown in Fig. 6. 

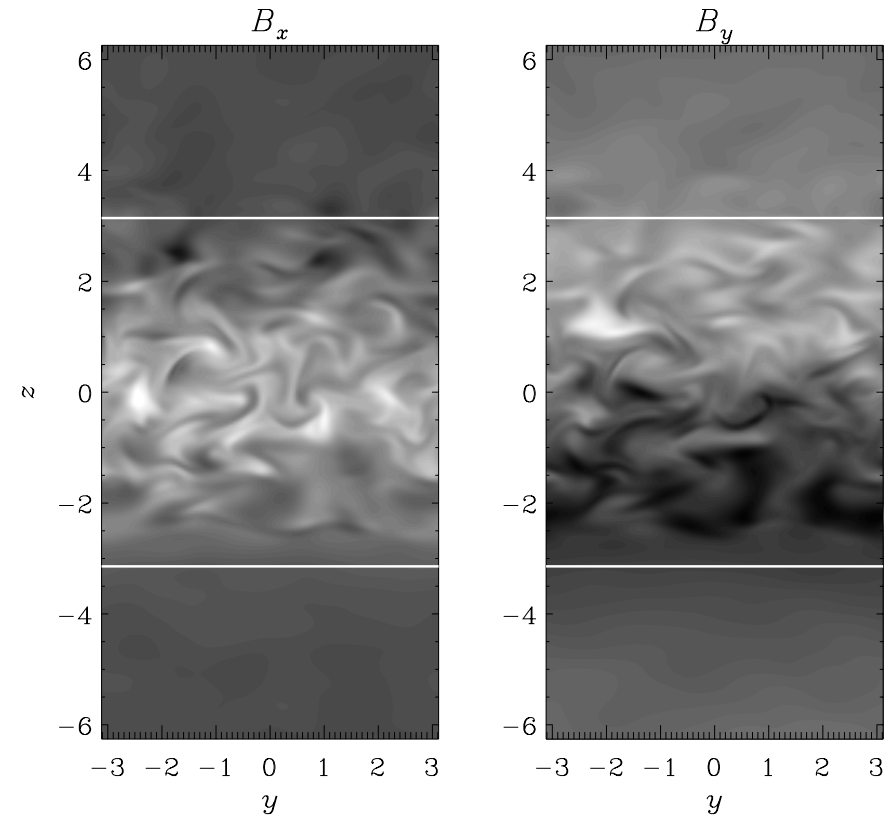

Fig. 5. Slices of the two horizontal field components for Buoy 2 with conducting halo and vertical gravity. The boundaries between halo and disc plane are indicated by white lines. $t=1400$. Dark and light shades indicate negative and positive values, respectively

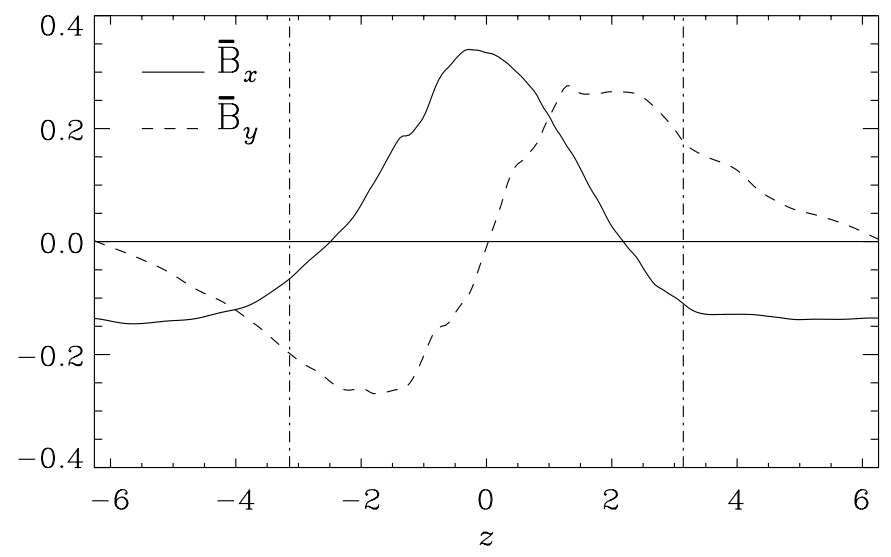

Fig. 6. Horizontal averages of $B_{x}$ and $B_{y}$ for Buoy 2. The boundary between halo and disc plane is indicated by the vertical dash-dotted lines. $t=1400$. Note that at this late time significant amounts of magnetic flux have been diffused into the halo

The evolution of kinetic and magnetic energies, as well as the magnetic energy of the large scale field, are shown in Fig. 7 for Buoy 2. In addition to a run starting with just a weak seed magnetic field we also show how the energies evolve when restarting from a snapshot of Buoy 1 at $t=800$.

In the following we study the results obtained in view of the helicity constraint, which was used extensively in B2001 in order to understand the slow growth of the large scale field and its final saturation level.

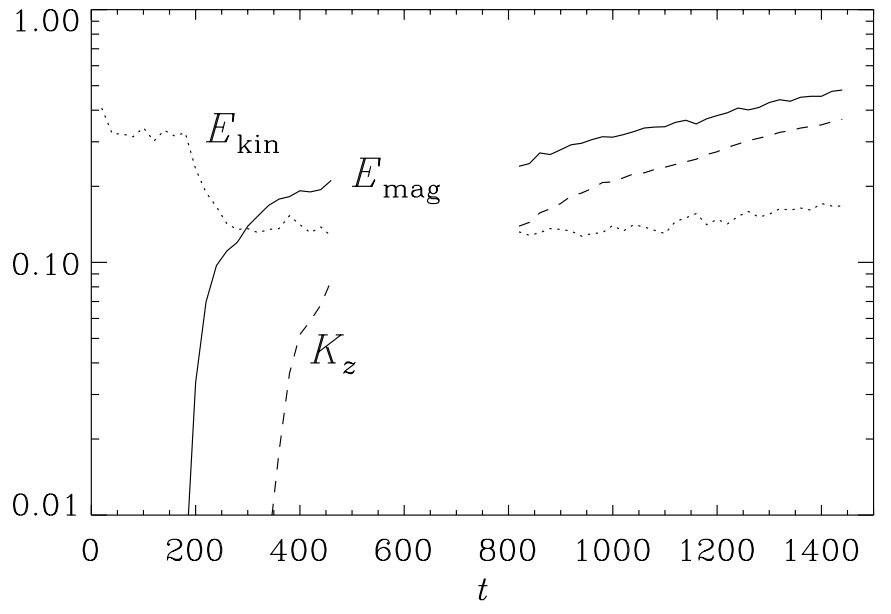

Fig. 7. Evolution of kinetic and magnetic energy together with the magnetic energies of the mean field. $\eta=10^{-3}$. Buoy 2 . The second part of the run $(t \geq 800)$ was obtained by restarting the simulation from Buoy 1

\section{Helicity constraint and helicity fluxes}

The resistively limited growth of large scale fields generated by helical turbulence is primarily a consequence of helicity conservation. In the present case, however, there is an additional surface term which results from the flux of helicity passing through those boundaries, so the equation of magnetic helicity conservation becomes

$\frac{\mathrm{d} H}{\mathrm{~d} t}=-2 \eta \mu_{0} C-Q$,

where $H$ and $C$ are magnetic and current helicities, respectively, and $Q$ is the surface-integrated helicity flux through the boundaries.

Owing to homogeneity of the system in the two horizontal directions we can consider integral quantities normalized per unit surface, so the current helicity (per unit surface) is therefore defined as

$C=\frac{1}{L_{x} L_{y}} \int \boldsymbol{J} \cdot \boldsymbol{B} \mathrm{d} V \equiv \int_{z_{1}}^{z_{2}} \overline{\boldsymbol{J} \cdot \boldsymbol{B}} \mathrm{d} z$,

where the overbar denotes $x$ and $y$ integration and division by the corresponding surface area $L_{x} L_{y}\left(=4 \pi^{2}\right)$, i.e. a horizontal average. The magnetic helicity would be $\int \overline{\boldsymbol{A} \cdot \boldsymbol{B}} \mathrm{d} z$, but in this form it is not gauge-invariant and would change if a constant or some gradient field were added to $\boldsymbol{A}$, which would leave $\boldsymbol{B}$ unchanged $^{1}$. The gauge invariant helicity of Berger \& Field (1984) is given by

$H=\int_{z_{1}}^{z_{2}} \overline{\left(\boldsymbol{A}+\boldsymbol{A}_{0}\right) \cdot\left(\boldsymbol{B}-\boldsymbol{B}_{0}\right)} \mathrm{d} z$,

where $\boldsymbol{B}_{0}=\boldsymbol{\nabla} \times \boldsymbol{A}_{0}$ is a potential field that satisfies

$\nabla^{2} \boldsymbol{A}_{0}=\mathbf{0}, \boldsymbol{\nabla} \cdot \boldsymbol{A}_{0}=0, A_{0 z}=0$ in $z_{1}<z<z_{2}$,

${ }^{1}$ We recall that in a periodic domain, $\int \boldsymbol{A} \cdot \boldsymbol{B} \mathrm{d} V$ is automatically gauge invariant, because then there are no surface terms and $\int \boldsymbol{\nabla} \phi \cdot \boldsymbol{B} \mathrm{d} V=-\int \phi \boldsymbol{\nabla} \cdot \boldsymbol{B} \mathrm{d} V=0$ for any $\phi$. 
and

$\boldsymbol{A}_{0}=\overline{\boldsymbol{A}}_{0}+\nabla_{\perp} \times(\psi \hat{\boldsymbol{z}}), \quad \nabla_{\perp}^{2} \psi=-B_{z}$ on $z=z_{1}, z_{2} ;$

here, $\nabla_{\perp}=\left(\partial_{x}, \partial_{y}, 0\right)$ is the horizontal nabla operator. Like $\boldsymbol{A}$, we assume $\boldsymbol{A}_{0}$ and $\psi$ to be periodic. This implies that the horizontal average of $\nabla_{\perp} \times(\psi \hat{\boldsymbol{z}})$ vanishes. This is the reason why we had to include an explicit $\overline{\boldsymbol{A}}_{0}$ term in Eq. (9) which is not needed by Berger \& Field (1984). Since the gauge-invariant magnetic helicity of the mean field plays an important role in the present context, we give an explicit derivation of this component of the magnetic helicity in Appendix A.

For the following analysis it is convenient to split the helicity terms into contributions from mean and fluctuating fields, $H=H_{\text {mean }}+H_{\text {fluct }}$, with (see Eq. (A.5))

$H_{\text {mean }}=\int_{z_{1}}^{z_{2}} \overline{\boldsymbol{A}} \cdot \overline{\boldsymbol{B}} \mathrm{d} z+\hat{\boldsymbol{z}} \cdot\left(\overline{\boldsymbol{A}}_{1} \times \overline{\boldsymbol{A}}_{2}\right)$,

where $\overline{\boldsymbol{A}}_{1}$ and $\overline{\boldsymbol{A}}_{2}$ are the values of $\overline{\boldsymbol{A}}$ at $z=z_{1}$ and $z_{2}$, respectively, and

$H_{\text {fluct }}=\int_{z_{1}}^{z_{2}} \overline{\left(\boldsymbol{a}+\boldsymbol{a}_{0}\right) \cdot\left(\boldsymbol{b}-\boldsymbol{b}_{0}\right)} \mathrm{d} z$.

The latter expression is just Eq. (7), but rewritten for the fluctuating fields, $\boldsymbol{a}=\boldsymbol{A}-\overline{\boldsymbol{A}}$, and correspondingly for the other variables. (We recall that Eq. (10) can also be written in a form similar to Eq. (11).) The components of the helicity flux, $Q=Q_{\text {mean }}+Q_{\text {fluct }}$, are given by (see Appendix B)

$Q_{\text {mean }}=-\left(\overline{\boldsymbol{E}}_{1}+\overline{\boldsymbol{E}}_{2}\right) \cdot \int_{z_{1}}^{z_{2}} \overline{\boldsymbol{B}} \mathrm{d} z$

and (see Berger \& Field 1984)

$Q_{\text {fluct }}=\left.2 \hat{\boldsymbol{z}} \cdot\left(\overline{\boldsymbol{e} \times \boldsymbol{a}_{0}}\right)\right|_{z_{1}} ^{z_{2}}$,

where

$\boldsymbol{E}=\eta \mu_{0} \boldsymbol{J}-\boldsymbol{u} \times \boldsymbol{B}$

is the electric field, and $\boldsymbol{E}=\overline{\boldsymbol{E}}+\boldsymbol{e}$ is its decomposition into mean and fluctuating components. In Appendix C we give the values of magnetic energy, magnetic helicity, integrated helicity flux, as well as the current helicity, for the eigenfunction of the $\alpha^{2}$-dynamo with the boundary condition $\bar{B}_{x}=\bar{B}_{y}=0$. Next we present in Fig. 8 the evolution of magnetic energy, magnetic and current helicities, and integrated helicity flux, for model Vert 4 . Note that, in the steady state, the helicity flux is balanced by the current helicity term, i.e.

$2 \eta \mu_{0} C_{\text {fluct }} \approx-Q_{\text {mean }}$.

The small scale current helicity term, $2 \eta \mu_{0} C_{\text {fluct }}$, may therefore be regarded as the source of magnetic helicity. Somewhat surprisingly, however, most of the helicity flux that is carried away results from the mean field, whilst most of the current helicity comes from the fluctuations. Similarly, $\left|H_{\text {mean }}\right| \gg\left|H_{\text {fluct }}\right|$. This dominance is
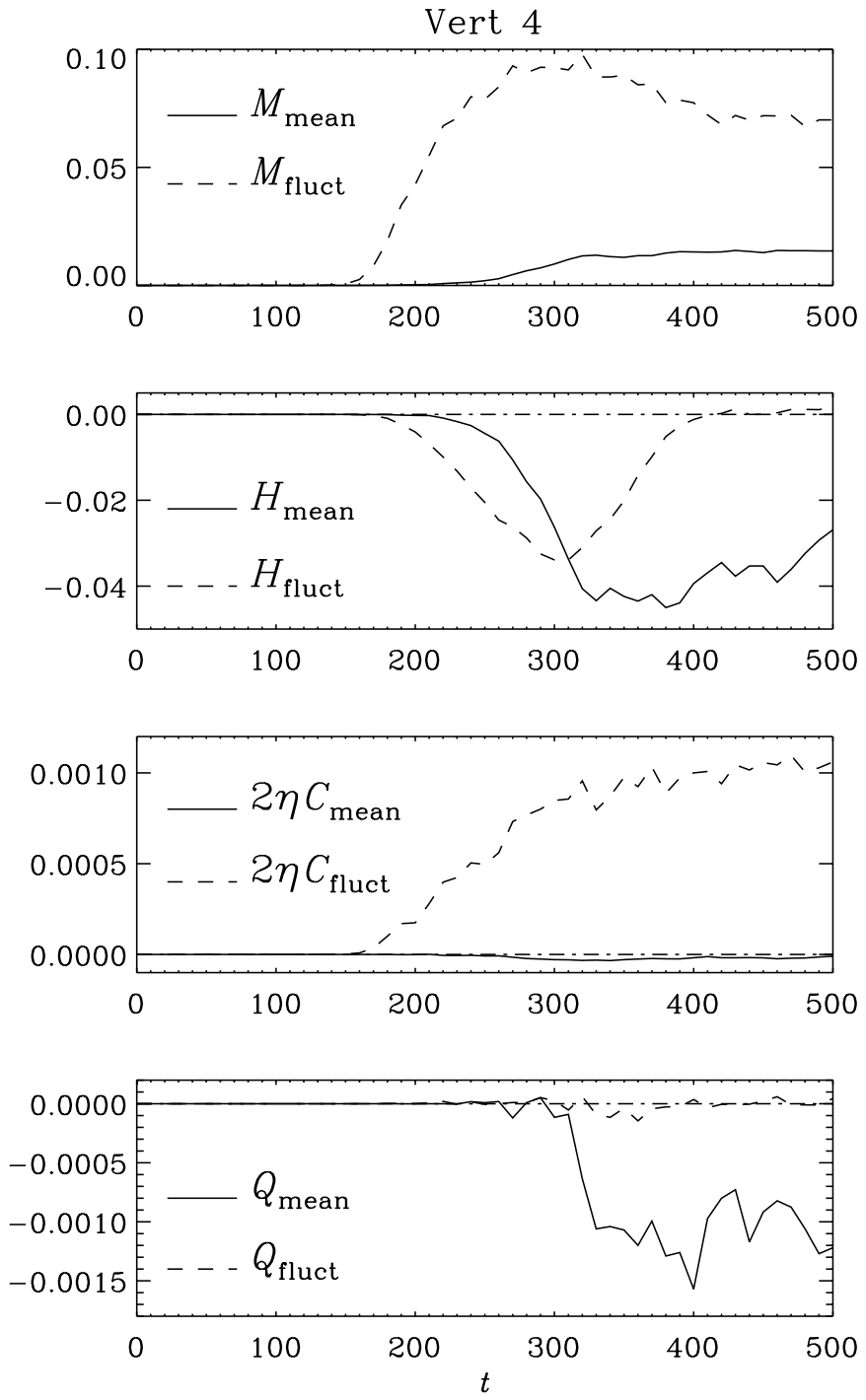

Fig. 8. Evolution of magnetic energy $M$, magnetic helicity $H$, current helicity $2 \eta C$ and magnetic helicity flux $Q$, for mean (solid lines) and fluctuating (dashed lines) field components for Vert 4 . ( $\mu_{0}=1$ factors are omitted in the legends)

partly explained by the factor $\left(k_{\mathrm{f}} / k_{1}\right)^{2}=25$ by which the large scales would exceed the small scales in the magnetic helicity relative to the current helicity [see B2001 after Eq. (41)].

It is then not too surprising, although perhaps somewhat disappointing, that almost all the magnetic helicity flux is in the large scales. We note, however, that the fractional contribution of small scale helicity flux does increase somewhat with increasing magnetic Reynolds number; see Table 2, where quantities relevant for the magnetic helicity equation are given for the different models with vertical field boundary condition. The fact that the $Q$ and $C$ terms in Eq. (15) do not balance exactly is partly explained by the finite value of $\mathrm{d} H / \mathrm{d} t$, even towards the end of the simulation. A potentially important feature is the negative burst of small-scale magnetic helicity around the time when the small scale field saturates $(t \approx 300$ in Figs. 8 and 9). In Halo 1 this is also associated with a 
Table 2. Comparison of relevant quantities in the magnetic helicity equation for different runs with vertical field boundary conditions

\begin{tabular}{lllcc}
\hline & Vert 1 & Vert 2 & Vert 3 & Vert 4 \\
\hline mesh & $30^{3}$ & $60^{3}$ & $120^{3}$ & $120^{3}$ \\
$\eta$ & 0.01 & 0.005 & 0.002 & 0.001 \\
$M_{\text {mean }}$ & 0.021 & 0.037 & 0.038 & 0.021 \\
$-H_{\text {mean }}$ & 0.051 & 0.069 & 0.084 & 0.037 \\
$2 \eta C_{\text {fluct }}$ & 0.0012 & 0.0023 & 0.0021 & 0.0012 \\
$-Q_{\text {mean }}$ & 0.0007 & 0.0017 & 0.0017 & 0.0010 \\
$M_{\text {fluct }} / M_{\text {mean }}$ & 0.3 & 0.7 & 1.6 & 3.2 \\
$H_{\text {fluct }} / H_{\text {mean }}$ & -0.03 & -0.09 & -0.12 & -0.23 \\
$C_{\text {fluct }} / C_{\text {mean }}$ & -4 & -13 & -26 & -69 \\
$Q_{\text {fluct }} / Q_{\text {mean }}$ & -0.000 & -0.003 & -0.005 & -0.007 \\
$\varepsilon_{H}$ & -2.5 & -1.9 & -2.2 & -1.8 \\
$\varepsilon_{C}$ & -0.7 & -0.5 & -0.5 & -0.4 \\
$\varepsilon_{Q}$ & -0.35 & -0.29 & -0.23 & -0.26 \\
\hline
\end{tabular}

similar negative burst in $Q_{\text {fluct }}$, as well as a with a period of enhanced small scale and large scale Poynting flux,

$P_{\text {fluct }}=\left.\hat{\boldsymbol{z}} \cdot(\overline{\boldsymbol{e} \times \boldsymbol{b}})\right|_{z_{1}} ^{z_{2}}, \quad$ and $\quad P_{\text {mean }}=\left.\hat{\boldsymbol{z}} \cdot(\overline{\boldsymbol{E}} \times \overline{\boldsymbol{B}})\right|_{z_{1}} ^{z_{2}},(16)$

respectively. (We recall that in the case of the vertical field boundary condition $P=0$ because $\boldsymbol{B}$ is vertical.)

Toward the end of the simulation with halo a significant mean field has been accumulated in the halo (see Fig. 6). This is a somewhat unrealistic feature that results from the use of periodic boundary conditions at the top of the halo. In reality the halo would extend further and the field would be lost into the exterior (i.e. interstellar or intergalactic) medium. An additional artifact of the halo being saturated with a large-scale field is the fact that at late times the Poynting flux is occasionally pointing inwards (Fig. 9), which is unlikely to occur in reality.

In Table 2 we also give the helicities and corresponding fluxes of the mean field in terms of the following nondimensional quantities:

$\varepsilon_{H}=\frac{k_{1} H_{\text {mean }} / \mu_{0}}{M_{\text {mean }}}$,

$\varepsilon_{C}=\frac{C_{\text {mean }} / k_{1}}{M_{\text {mean }}}$

$\varepsilon_{Q}=\frac{Q_{\text {mean }} / \mu_{0}}{u_{\mathrm{rms}} M_{\text {mean }}}$

where $k_{1}=2 \pi / L$ is the smallest wavenumber in the domain (and usually the wavenumber of the large scale field); in our case $k_{1}=1$. We also have $\mu_{0}=1$, but we keep this factor in some of the expressions for clarity.

Table 3 gives the same quantities for the model with a conducting halo. Here the overall helicity flux is generally somewhat smaller than in the case with vertical field boundary conditions. However, the fractional contribution of small scale helicity flux is larger. The magnetic helicity production, which is dominated by $2 \eta \mu_{0} C_{\text {fluct }}$, is still
Table 3. Comparison of relevant quantities in the magnetic helicity equation for different runs with different implementations of a conducting halo. Buoy 1 has a poorly conducting halo, whereas Buoy 2 has a halo conductivity equal to the disc conductivity. In Halo 1 the value of $\eta$ was so small that the full saturation field strength has not yet been achieved by the end of the simulation. The asterisks mark those quantities that are likely to change if the run was continued further. In all cases we use $120 \times 120 \times 240$ mesh points

\begin{tabular}{lllcc}
\hline & Halo 1 & Halo 2 & Buoy 1 & Buoy 2 \\
\hline$\eta$ & 0.0005 & 0.001 & 0.001 & 0.001 \\
$\eta_{\text {halo }}$ & 0.0205 & 0.021 & 0.021 & 0.001 \\
$M_{\text {mean }}$ & $0.052^{*}$ & 0.092 & 0.15 & 0.26 \\
$-H_{\text {mean }}$ & $0.170^{*}$ & 0.300 & 0.53 & 0.94 \\
$2 \eta C_{\text {fluct }}$ & 0.0007 & 0.0012 & 0.0019 & 0.0021 \\
$-Q_{\text {mean }}$ & 0.0000 & 0.0000 & 0.0007 & 0.0009 \\
$P_{\text {mean }}$ & 0.0000 & 0.0000 & 0.0003 & 0.0003 \\
$M_{\text {fluct }} / M_{\text {mean }}$ & 1.3 & 0.7 & 0.6 & 0.4 \\
$H_{\text {fluct }} / H_{\text {mean }}$ & -0.06 & -0.04 & -0.03 & -0.02 \\
$C_{\text {fluct }} / C_{\text {mean }}$ & -10 & -4.8 & -4.4 & -3.1 \\
$Q_{\text {fluct }} / Q_{\text {mean }}$ & $+0.27^{*}$ & -0.09 & +0.006 & -0.034 \\
$\varepsilon_{H}$ & -3.3 & -3.3 & -3.5 & -3.6 \\
$\varepsilon_{C}$ & -1.3 & -1.3 & -1.4 & -1.3 \\
$\varepsilon_{Q}$ & -0.002 & +0.005 & -0.036 & -0.022 \\
\hline
\end{tabular}

similar in the two cases. In the following section we discuss why the losses of the field with large scale helicity significantly lower the saturation field strength.

\section{Interpretation}

\subsection{The helicity constraint}

In B2001 it was possible to understand the magnetic field evolution as a consequence of the magnetic helicity evolution. The current data show that most of the magnetic helicity and its corresponding flux is in the mean field. Thus, helicity conservation is governed approximately by

$\frac{\mathrm{d}}{\mathrm{d} t} H_{\text {mean }} \approx-2 \eta \mu_{0}\left(C_{\text {mean }}+C_{\text {fluct }}\right)-Q_{\text {mean }}$.

Furthermore, in B2001 it was found that for strongly helical fields both current and magnetic helicities are proportional to the large scale magnetic energy. The same is true here: from Table 2 we see that the ratio $\varepsilon_{H}$ is around 2 , and that $\varepsilon_{C}$ is around $0.5-1$, We also note that the helicity flux is a certain fraction of the magnetic energy, i.e. $\varepsilon_{Q} \approx 0.2-0.4$. Using the fact that all these ratios are approximately constant for the different runs, we may write

$\frac{\mathrm{d}}{\mathrm{d} t} M_{\text {mean }} \approx-2 \eta_{\mathrm{eff}} k_{1}^{2} M_{\text {mean }}+2 \eta k_{1} \frac{C_{\text {fluct }}}{\varepsilon_{H}}$,

where we have defined an effective magnetic diffusion coefficient via

$2 \eta_{\mathrm{eff}}=\left(2 \eta \varepsilon_{C}+\varepsilon_{Q} k_{1}^{-1} u_{\mathrm{rms}}\right) / \varepsilon_{H}$. 
Halo 1
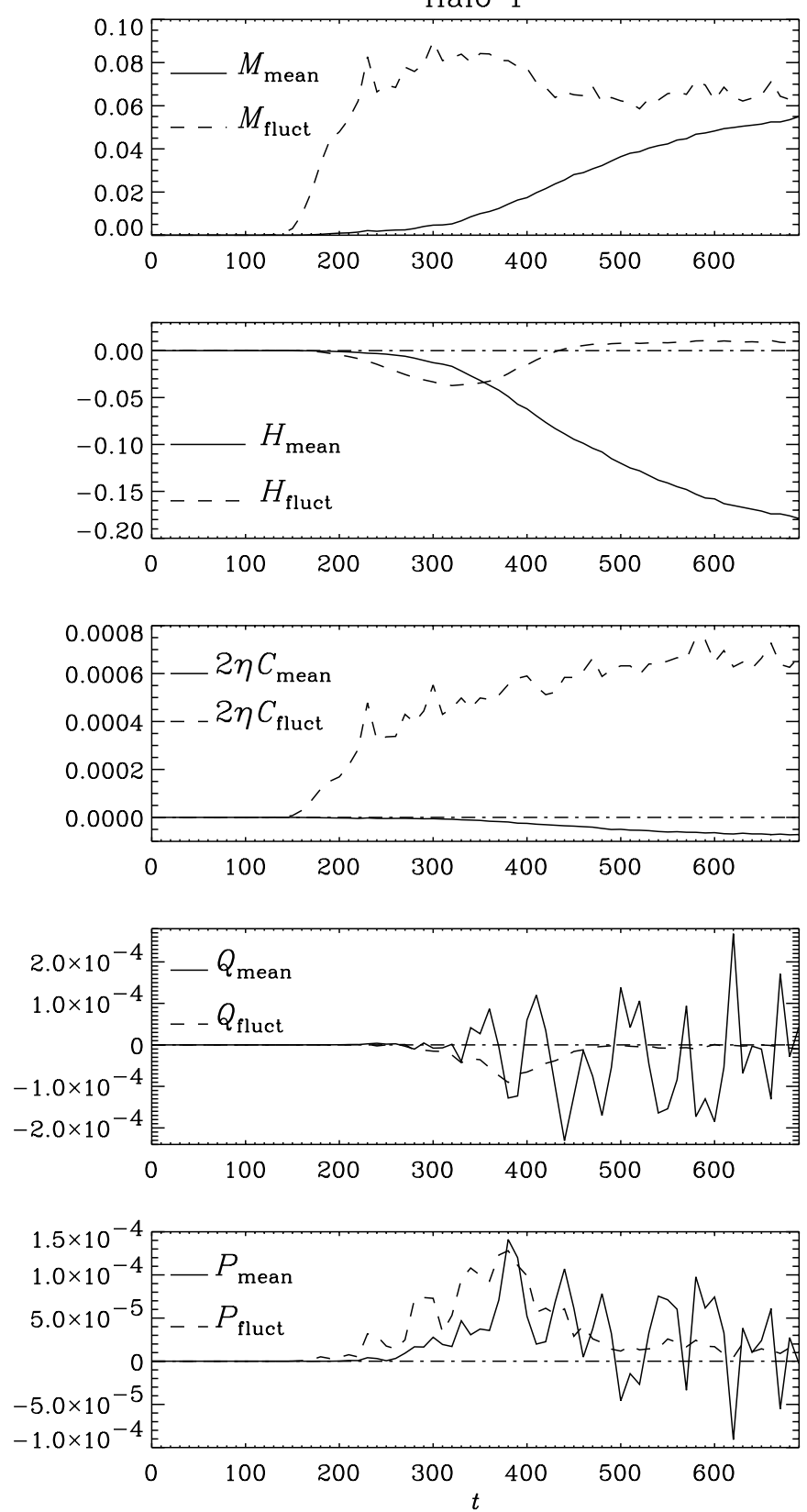

Fig. 9. Evolution of magnetic energy $M$, magnetic helicity $H$, current helicity $2 \eta C$, magnetic helicity flux $Q$, and magnetic energy (or Poynting) flux $P$, for mean (solid lines) and fluctuating (dashed lines) field components for Halo 1. All quantities are evaluated between the boundaries $z_{1}=-\pi$ and $z_{2}=\pi$. After $t \approx 400-500$ the field in the halo becomes noticeable and effects of the periodic boundaries in the $z$-direction begin to affect the evolution of the fluxes

Equation (21) has the solution

$M_{\text {mean }} \approx \frac{\eta}{\eta_{\text {eff }}} \frac{C_{\text {fluct }}}{\varepsilon_{H} k_{1}}\left[1-\mathrm{e}^{-2 \eta_{\text {eff }} k_{1}^{2}\left(t-t_{\mathrm{s}}\right)}\right]$ for $t>t_{\mathrm{s}}$,

where $t_{\mathrm{s}}=-\lambda \ln \left(B_{\text {ini }} / B_{\text {eq }}\right)$ is the saturation time of the total (mainly small scale) field, $\lambda$ is the kinematic growth rate of the dynamo, and $B_{\text {ini }}$ is the initial field strength. Equation (23) is analogous to Eq. (45) of B2001, but since $\eta_{\text {eff }}$ is much larger than $\eta$, the time scale for reaching full saturation is reduced from $\left(\eta k_{1}^{2}\right)^{-1}$ to $\left(\eta_{\text {eff }} k_{1}^{2}\right)^{-1}$ due to the presence of boundaries. However, this result is obtained at the price of having reduced the saturation field amplitude. Therefore, all this flux term really does is cutting off the growth of the field earlier than otherwise, so at any time the energy is less than in the case of a periodic domain. Indeed, expanding the exponential for short enough times we find that

$M_{\text {mean }} \approx 2 \eta k_{1} \frac{C_{\text {fluct }}}{\varepsilon_{H}}\left(t-t_{\mathrm{s}}\right)$ for $t>t_{\mathrm{s}}$,

which shows that the initial growth time is still inversely proportional to the microscopic magnetic diffusivity.

There is not much scope for increasing the rate of large-scale field production in Eq. (24). The value of $C_{\text {fluct }}$ does not strongly depend on the details of the model (cf. Tables 2 and 3 ). The only way out seems to be in lowering the value of $\varepsilon_{H}$, which corresponds to making the field less helical. This is what happened when adding the effects of shear (Brandenburg et al. 2001).

If $\eta / \eta_{\text {eff }}$ becomes very small the estimates given in Eqs. (23) and (24) underestimate the value of $M_{\text {mean }}$, because there is always some mean field that results from imperfect cancellations of the fluctuating field at the energy carrying wavenumber which is here $k_{\mathrm{f}}$. The resulting energy of the mean field would be of the order $\left(k_{1} / k_{\mathrm{f}}\right)^{3} M_{\text {fluct }}$. In the present case we are however far away from this lower limit.

In the presence of a conducting halo the helicity flux is smaller than in the case of the vertical field boundary condition; see Table 3 and Fig. 9 where we also show, in addition to magnetic helicity flux and current helicity, the evolution of magnetic energy, magnetic helicity, and the Poynting flux. Note in particular that $Q_{\text {mean now fluctu- }}$ ates about zero. Consequently, there is no longer an approximate balance between $2 \eta C_{\text {fluct }}$ and $Q_{\text {mean }}$. Instead, the $C_{\text {mean }}$ term becomes more important and would, in the limit of a periodic domain $(Q \rightarrow 0)$, balance $C_{\text {fluct }}$, as was found in the case of periodic domains; see B2001.

\subsection{Comparison with a mean-field model}

In the case of a periodic domain it was possible to reproduce the slow saturation of the magnetic energy with an $\alpha^{2}$-dynamo with simultaneous $\alpha$ and $\eta_{\mathrm{t}}$-quenching of the form

$\alpha=\frac{\alpha_{0}}{1+\alpha_{B} \overline{\boldsymbol{B}}^{2} / B_{\mathrm{eq}}^{2}}, \quad \eta_{\mathrm{t}}=\frac{\eta_{\mathrm{t} 0}}{1+\eta_{B} \overline{\boldsymbol{B}}^{2} / B_{\mathrm{eq}}^{2}}$,

where $\alpha_{B}=\eta_{B}$ was assumed, and both quantities were proportional to the magnetic Reynolds number, as was suggested by Vainshtein \& Cattaneo (1992). The reason this worked was because of the presence of microscopic magnetic diffusion which was not quenched, and which led to saturation such that (see Eqs. (55) and (56) of B2001) $\alpha_{B} \frac{B_{\mathrm{fin}}^{2}}{B_{\mathrm{eq}}^{2}} \approx \frac{\lambda}{\eta k_{1}^{2}} \approx 0.01 R_{\mathrm{m}} \quad$ (for a periodic domain), 


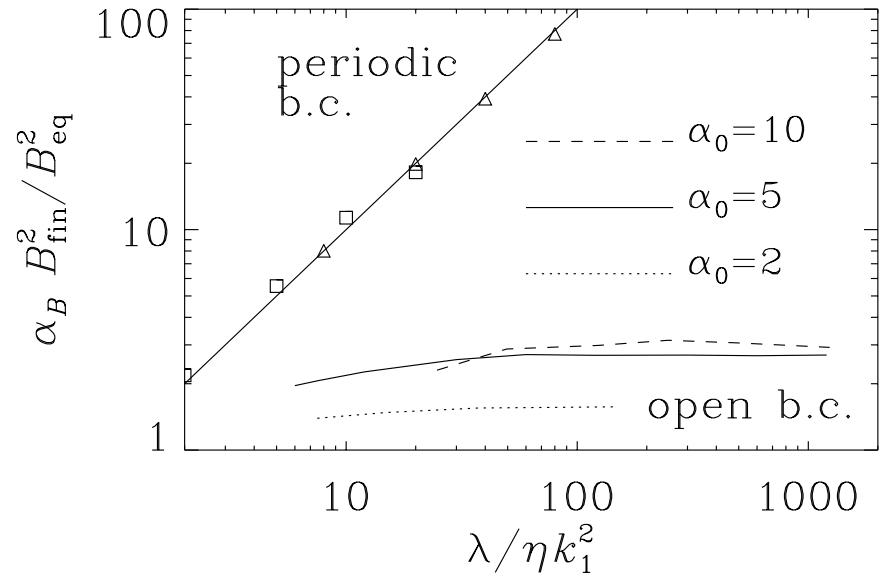

Fig. 10. Normalized magnetic energy in one-dimensional mean-field models with periodic and open boundary conditions. The models with open boundaries allow for helicity flux out of the domain and yield significantly smaller field amplitudes when the magnetic Reynolds number, or $\lambda / \eta k_{1}^{2}$, is large. In the periodic case the growth rate is $\lambda=\alpha-\eta_{\mathrm{T}}$, whilst in the case with open boundaries $\lambda=\frac{1}{4}\left(\alpha^{2}-\eta_{\mathrm{T}}^{2}\right) / \eta_{\mathrm{T}}$. For the periodic boundary condition, squares correspond to $\alpha_{0}=2$ and triangles to $\alpha_{0}=5$. The data are obtained numerically using a time-stepping method

where $B_{\text {fin }}$ is the final magnitude of the mean field, $\lambda=$ $\alpha k_{1}-\eta_{\mathrm{T}} k_{1}^{2}$ is the kinematic growth rate of the dynamo, $\eta_{\mathrm{T}}=\eta+\eta_{\mathrm{t}}$ is the total magnetic diffusivity, and $R_{\mathrm{m}}=$ $u_{\mathrm{rms}} L / \eta$ is the magnetic Reynolds number with respect to the scale of the domain.

In the present case of open boundaries there is an additional loss term and so Eq. (26) no longer holds. A numerical integration of the nonlinear $\alpha^{2}$-dynamo equations with boundary conditions $\bar{B}_{x}=\bar{B}_{y}=0$ (Meinel \& Brandenburg 1990) shows that the term $\alpha_{B} B_{\text {fin }}^{2} / B_{\text {eq }}^{2}$ saturates at a value of around 3 and is only weakly dependent on the value of $\alpha_{0}$; see Fig. 10 , where $\eta+\eta_{\mathrm{t} 0}=1$. In the simulations, on the other hand, the final large-scale field strength decreases with $R_{\mathrm{m}}$ like $R_{\mathrm{m}}^{-1 / 2}$, even though the total (mainly small scale) magnetic field strength is always close to the equipartition field strength; see Fig. 4 and Table 1 . From this we can conclude that $\alpha_{B}$ increases only like $R_{\mathrm{m}}^{1 / 2}$. This is to be contrasted with the case of periodic domains where $B_{\text {fin }} / B_{\text {eq }}$ was independent of $R_{\mathrm{m}}$ and therefore $\alpha_{B} \propto R_{\mathrm{m}}$. It is important to realize, however, that the scaling of $\alpha_{B}$ with $R_{\mathrm{m}}$ is secondary to having strong large-scale dynamo action. What really matters is the saturation level and the time scale on which full saturation is reached. These properties are controlled primarily by the magnetic helicity equation which, in turn, is sensitive to boundary conditions, anisotropies, and the presence of shear, for example (e.g. Brandenburg et al. 2001).

\section{Conclusions}

The results presented in this paper show that open boundary conditions, and boundaries different from periodic ones, can have profound effects on the large-scale field generation in helical flows. Firstly, unlike the case of periodic domains, a strictly force-free large-scale magnetic field is no longer possible. Secondly, the loss of magnetic helicity through the boundaries severely limits the large-scale field amplitude attainable in simulations. This is quite different from the case of periodic domains, where the large-scale field energy can exceed the kinetic energy, provided there is sufficient scale separation between the energy carrying scale and the scale of the system (see Sects. 3.5 and 3.6 of B2001). For an open simulation volume with a vertical field boundary condition, however, the large-scale field amplitude can be severely limited in a magnetic Reynolds number $\left(R_{\mathrm{m}}\right)$ dependent fashion. Again, this result can be modelled with a mean-field $\alpha^{2}$-dynamo, where the $\alpha$-effect and turbulent magnetic diffusivity are quenched. However, the $R_{\mathrm{m}}$-dependence of these quenching expressions is here less extreme than in the case of periodic domains where the field can be force-free (B2001). This suggests that a dependence of $\alpha$ and $\eta_{\mathrm{t}}$ on $\overline{\boldsymbol{B}}^{2}$ alone is too simplistic, and that there might be an additional dependence on $\overline{\boldsymbol{J}} \times \overline{\boldsymbol{B}}$ or $\overline{\boldsymbol{J}} \cdot \overline{\boldsymbol{B}}$, for example, that would react specifically on non force-free magnetic fields.

Our result that the quenching of $\alpha$ and $\eta_{\mathrm{t}}$ can be sensitive to external factors is not entirely unfamiliar. For example in the presence of large scale shear (corresponding to differential rotation) the quenching of $\alpha$ and $\eta_{\mathrm{t}}$ was already found to be significantly reduced; see Brandenburg et al. (2001). A significant reduction of the quenching may explain why the cycle period in oscillatory dynamos does not need to scale linearly with the resistive time scale (which would be too long). Nevertheless, the growth time of a large-scale dynamo with helicity does seem to scale linearly with the resistive time scale in all cases investigated so far.

In the simulations presented here, the effects of open boundaries and magnetic buoyancy were too weak to produce any sizeable small scale magnetic helicity fluxes. However, this leaves unanswered the question whether small scale magnetic helicity fluxes are in principle capable of reducing the long time scales that result from magnetic helicity conservation. In order to clarify this one may need to resort to models where small-scale flux losses are somehow artificially enhanced.

Finally, it should be mentioned that we have here neglected the effects of an equator where the sign of the helicity would change. In the present framework this can be modelled by modulating the sign of the helicity of the forcing function in the $z$-direction. Preliminary results have been reported in Brandenburg (2001b), but the results are quite similar to those in the case of open boundary conditions. Indeed an estimate similar to Eq. (23) has been obtained for the resulting magnetic energy of the mean field.

It is now important to move on to more realistic global simulations which would automatically allow for helicity exchange across the equator and produce flux losses through winds and eruptions into the corona. It is indeed 
quite possible that significant helicity fluxes are only produced by the combined action of stratification and shear (Berger \& Ruzmaikin 2000; Vishniac \& Cho 2000), which again would automatically be addressed by more realistic global simulations. Even if the magnetic helicity flux continues to be dominated by large scale contributions, it could be argued that such an effect could alleviate the helicity constraint, because saturation (albeit at a lower value) does occur earlier. It would seem necessary, however, to compensate those additional losses by more powerful dynamo action. Again, the effects of shear may prove important, because it provides additional toroidal field that is non-helical and hence not subject to any constraint.

Acknowledgements. We thank Eric Blackman and Anvar Shukurov for critical comments and suggestions that have improved the presentation of the results. This work was partially supported by PPARC (Grant PPA/G/S/1997/00284) and the Leverhulme Trust (Grant F/125/AL). Use of the PPARC supported supercomputers in St Andrews and Leicester is acknowledged.

\section{Appendix A: Gauge-independent magnetic helicity of the mean field}

In a periodic domain the magnetic helicity is gauge invariant, so $\overline{\boldsymbol{A}} \cdot \overline{\boldsymbol{B}}$ (in $z_{1} \leq z<z_{2}$ ) can be made gauge invariant by linearly extrapolating $\overline{\boldsymbol{A}}$ from $z_{2}$ to $z_{3}$, where $\overline{\boldsymbol{A}}_{3}=\overline{\boldsymbol{A}}_{1}$ is assumed, and $\overline{\boldsymbol{A}}_{i}$ are the values of $\overline{\boldsymbol{A}}$ at $z=z_{i}$, where $i=1$, 2 , or 3 . Linear extrapolation is used because this corresponds to the simplest possible, current-free field. Thus, we can write for the magnetic helicity of the mean field

$H_{\text {mean }}=\int_{z_{1}}^{z_{2}} \overline{\boldsymbol{A}} \cdot \overline{\boldsymbol{B}} \mathrm{d} z+\int_{z_{2}}^{z_{3}} \tilde{\boldsymbol{A}} \cdot \tilde{\boldsymbol{B}} \mathrm{d} z$,

where

$\tilde{\boldsymbol{A}}=\overline{\boldsymbol{A}}_{2}+\frac{\overline{\boldsymbol{A}}_{3}-\overline{\boldsymbol{A}}_{2}}{z_{3}-z_{2}}\left(z-z_{2}\right)$

is the linear extension of $\overline{\boldsymbol{A}}$ from $z_{2}$ to $z_{3}$, and

$\tilde{\boldsymbol{B}}=\boldsymbol{\nabla} \times \tilde{\boldsymbol{A}}=\hat{\boldsymbol{z}} \times \frac{\overline{\boldsymbol{A}}_{3}-\overline{\boldsymbol{A}}_{2}}{z_{3}-z_{2}}$,

so

$\tilde{\boldsymbol{A}} \cdot \tilde{\boldsymbol{B}}=\overline{\boldsymbol{A}}_{2} \cdot\left[\hat{\boldsymbol{z}} \times \frac{\overline{\boldsymbol{A}}_{3}-\overline{\boldsymbol{A}}_{2}}{z_{3}-z_{2}}\right]=\hat{\boldsymbol{z}} \cdot \frac{\overline{\boldsymbol{A}}_{3} \times \overline{\boldsymbol{A}}_{2}}{z_{3}-z_{2}}$.

Since $\overline{\boldsymbol{A}}_{3}=\overline{\boldsymbol{A}}_{1}$ we have

$\int_{z_{2}}^{z_{3}} \tilde{\boldsymbol{A}} \cdot \tilde{\boldsymbol{B}} \mathrm{d} z=\hat{\boldsymbol{z}} \cdot\left(\overline{\boldsymbol{A}}_{1} \times \overline{\boldsymbol{A}}_{2}\right)$,

which is independent of the choice of the value of $z_{3}$. In particular, we may choose $z_{3}=z_{1}$, in which case this definition is identical to Eq. (7) with Eq. (9) and $\overline{\boldsymbol{A}}_{0}=\tilde{\boldsymbol{A}}$.

\section{Appendix B: Helicity flux of the mean field}

The evolution of the gauge-invariant magnetic helicity of the mean field is given by

$$
\begin{array}{r}
\frac{\mathrm{d}}{\mathrm{d} t} H_{\text {mean }}=\int_{z_{1}}^{z_{2}}(\dot{\overline{\boldsymbol{A}}} \cdot \overline{\boldsymbol{B}}+\overline{\boldsymbol{A}} \cdot \dot{\overline{\boldsymbol{B}}}) \mathrm{d} z \\
+\hat{\boldsymbol{z}} \cdot\left(\dot{\overline{\boldsymbol{A}}}_{1} \times \overline{\boldsymbol{A}}_{2}+\overline{\boldsymbol{A}}_{1} \times \dot{\overline{\boldsymbol{A}}}_{2}\right)
\end{array}
$$

where dots denote partial time derivatives, but, because $\dot{\overline{\boldsymbol{A}}}=-\overline{\boldsymbol{E}}$ in our gauge (cf. Eq. (3)), we have

$$
\begin{array}{r}
\frac{\mathrm{d}}{\mathrm{d} t} H_{\text {mean }}=-2 \int_{z_{1}}^{z_{2}} \overline{\boldsymbol{E}} \cdot \overline{\boldsymbol{B}} \mathrm{d} z-\left.\hat{\boldsymbol{z}} \cdot(\overline{\boldsymbol{E}} \times \overline{\boldsymbol{A}})\right|_{z_{1}} ^{z_{2}} \\
-\hat{\boldsymbol{z}} \cdot\left(\overline{\boldsymbol{E}}_{1} \times \overline{\boldsymbol{A}}_{2}+\overline{\boldsymbol{A}}_{1} \times \overline{\boldsymbol{E}}_{2}\right)
\end{array}
$$

The last two terms reduce to

$-\hat{\boldsymbol{z}} \cdot\left[\left(\overline{\boldsymbol{E}}_{1}+\overline{\boldsymbol{E}}_{2}\right) \times\left(\overline{\boldsymbol{A}}_{2}-\overline{\boldsymbol{A}}_{1}\right)\right]$,

which can also be written as $\left(\overline{\boldsymbol{E}}_{1}+\overline{\boldsymbol{E}}_{2}\right) \cdot \int_{z_{1}}^{z_{2}} \overline{\boldsymbol{B}} \mathrm{d} z$.

\section{Appendix C: Helicity flux for the eigenfunction of the associated $\alpha^{2}$-dynamo}

The kinematic $\alpha^{2}$-dynamo equation with $\overline{\boldsymbol{B}}=\overline{\boldsymbol{B}}(z, t)=$ $\left(\bar{B}_{x}, \bar{B}_{y}, 0\right)$ can be written in the form

$\partial_{t} \mathcal{B}=\mathrm{i} \alpha \partial_{z} \mathcal{B}+\eta_{\mathrm{T}} \partial_{z}^{2} \mathcal{B}$

where we have adopted complex notation, $\mathcal{B}=\bar{B}_{x}+\mathrm{i} \bar{B}_{y}$, and $\alpha$ and $\eta_{\mathrm{T}}$ are assumed constant. The eigenfunction that satisfies $\mathcal{B}=0$ at $z= \pm \pi$ is (e.g., Meinel \& Brandenburg 1990)

$\mathcal{B}(z, t)=\mathcal{B}_{0} \cos \frac{z}{2} \exp \left(\lambda t-\frac{\alpha}{\eta_{\mathrm{T}}} \frac{\mathrm{i} z}{2}\right)$

where $\mathcal{B}_{0}$ is a complex constant and $\lambda=\frac{1}{4}\left(\alpha^{2}-\eta_{\mathrm{T}}^{2}\right) / \eta_{\mathrm{T}}$ is the growth rate. We are interested in the marginally excited mode, $\lambda=0$, and so we assume for simplicity $\mathcal{B}_{0}=\alpha=\eta_{\mathrm{T}}=1$, so we have

$\mathcal{B}(z, t)=\mathrm{e}^{-\mathrm{i} z / 2} \cos (z / 2)$.

The complex current density, $\mathcal{J}=\mathrm{i} \partial_{z} \mathcal{B}$, is given by

$\mathcal{J}(z, t)=\frac{1}{2} \exp (-\mathrm{i} z)$

and the complex vector potential is, apart from some arbitrary constant,

$\mathcal{A}(z, t)=\frac{1}{2} \exp (-\mathrm{i} z)-\frac{1}{2} \mathrm{i} z$

which satisfies $\partial_{z}^{2} \mathcal{A}=-\mathcal{J}$ and $\mathcal{B}=\mathrm{i} \partial_{z} \mathcal{A}$. For this field the magnetic energy $M_{\text {mean }}$, the magnetic and current helicities $H_{\text {mean }}$ and $C_{\text {mean }}$, as well as the integrated magnetic helicity flux $Q_{\text {mean, }}$, are given by

$M_{\text {mean }}=\frac{\pi}{2}=C_{\text {mean }}, \quad H_{\text {mean }}=\frac{3 \pi}{2}, \quad Q_{\text {mean }}=\pi$. 
The Lorentz force is $\overline{\boldsymbol{J}} \times \overline{\boldsymbol{B}}=\left(0,0, \frac{1}{4} \sin z\right)$, and the current helicity density is $\overline{\boldsymbol{J}} \cdot \overline{\boldsymbol{B}}=\frac{1}{2} \cos ^{2}(z / 2)$, so

$$
\frac{\left\langle(\overline{\boldsymbol{J}} \times \overline{\boldsymbol{B}})^{2}\right\rangle}{\left\langle\overline{\boldsymbol{J}}^{2}\right\rangle\left\langle\overline{\boldsymbol{B}}^{2}\right\rangle}=\frac{1}{4}, \quad \frac{\left\langle(\overline{\boldsymbol{J}} \cdot \overline{\boldsymbol{B}})^{2}\right\rangle}{\left\langle\overline{\boldsymbol{J}}^{2}\right\rangle\left\langle\overline{\boldsymbol{B}}^{2}\right\rangle}=\frac{3}{4},
$$

so the field is neither fully force-free nor fully helical, but something in between. (For the mean fields of B2001 those values were 0 and 1 , respectively.)

\section{References}

Berger, M., \& Field, G. B. 1984, JFM, 147, 133

Berger, M. A., \& Ruzmaikin, A. 2000, JGR, 105, 10481

Blackman, E. G., \& Field, G. F. 2000a, ApJ, 534, 984

Blackman, E. G., \& Field, G. F. 2000b, Phys. Plasmas, submitted [astro-ph/0012048]

Brandenburg, A. 2001a, ApJ, 550, April 1 issue, [astro-ph/0006186] (B2001)
Brandenburg, A. 2001b, in Dynamo and dynamics, a mathematical challenge, ed. P. Chossat (Nato ASI Series, Kluwer Publ., in press) [astro-ph/0012112]

Brandenburg, A., Bigazzi, A., \& Subramanian, K. 2001, MNRAS, submitted [astro-ph/0011081]

Brandenburg, A., Nordlund, Å., Stein, R. F., \& Torkelsson, U. 1995, ApJ, 446, 741

Cattaneo, F., \& Hughes, D. W. 1996, Phys. Rev. E, 54, R4532

Hurlburt, N. E., \& Toomre, J. 1988, ApJ, 327, 920

Kleeorin, N. I, Moss, D., Rogachevskii, I., \& Sokoloff, D. 2000, A\&A, 361, L5

Krause, F., \& Rädler, K.-H. 1980, Mean-Field Magnetohydrodynamics and Dynamo Theory (Pergamon Press, Oxford)

Meinel, R., \& Brandenburg, A. 1990, A\&A, 238, 369

Moffatt, H. K. 1978, Magnetic Field Generation in Electrically Conducting Fluids (CUP, Cambridge)

Seehafer, N. 1996, Phys. Rev. E, 53, 1283

Vainshtein, S. I., \& Cattaneo, F. 1992, ApJ, 393, 165

Vishniac, E. T., \& Cho, J. 2000, ApJ, in press [astro-ph/0010373] 